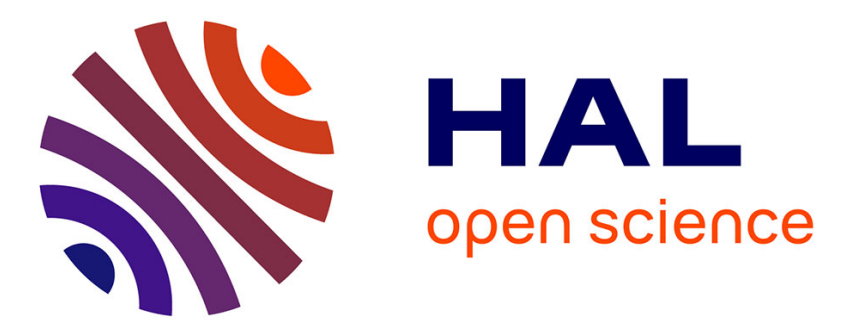

\title{
High Frequency Monitoring Reveals Aftershocks in Subcritical Crack Growth
}

\author{
Marija Stojanova, S. Santucci, L. Vanel, O. Ramos
}

\section{To cite this version:}

Marija Stojanova, S. Santucci, L. Vanel, O. Ramos. High Frequency Monitoring Reveals Aftershocks in Subcritical Crack Growth. Physical Review Letters, 2014, 112 (11), 10.1103/PhysRevLett.112.115502 . hal-01727333v1

\section{HAL Id: hal-01727333 https://univ-lyon1.hal.science/hal-01727333v1}

Submitted on 12 Mar 2018 (v1), last revised 16 Mar 2018 (v2)

HAL is a multi-disciplinary open access archive for the deposit and dissemination of scientific research documents, whether they are published or not. The documents may come from teaching and research institutions in France or abroad, or from public or private research centers.
L'archive ouverte pluridisciplinaire HAL, est destinée au dépôt et à la diffusion de documents scientifiques de niveau recherche, publiés ou non, émanant des établissements d'enseignement et de recherche français ou étrangers, des laboratoires publics ou privés. 


\title{
High Frequency Monitoring Reveals Aftershocks in Subcritical Crack Growth
}

\author{
M. Stojanova, ${ }^{1}$ S. Santucci, ${ }^{2}$ L. Vanel, ${ }^{1}$ and O. Ramos ${ }^{1, *}$ \\ ${ }^{1}$ Institut Lumière Matière, UMR5306 Université Lyon 1-CNRS, Université de Lyon, 69622 Villeurbanne, France \\ ${ }^{2}$ Laboratoire de Physique, CNRS UMR 5672, Ecole Normale Supérieure de Lyon, Université de Lyon, \\ 46 allée d'Italie, 69364 Lyon Cedex 07, France
}

(Received 9 July 2013; published 18 March 2014)

\begin{abstract}
By combining direct imaging and acoustic emission measurements, the subcritical propagation of a crack in a heterogeneous material is analyzed. Both methods show that the fracture proceeds through a succession of discrete events. However, the macroscopic opening of the fracture captured by the images results from the accumulation of more-elementary events detected by the acoustics. When the acoustic energy is cumulated over large time scales corresponding to the image acquisition rate, a similar statistics is recovered. High frequency acoustic monitoring reveals aftershocks responsible for a time scale dependent exponent of the power law energy distributions. On the contrary, direct imaging, which is unable to resolve these aftershocks, delivers a misleading exponent value.
\end{abstract}

Introduction.-Industrial and architectural designs evolve continuously towards thinner and lighter structures, many of them subjected to a permanent subcritical stress. As stresses intensify around a flaw in the material [1], a micro-crack can eventually start growing, until it reaches a critical length where the whole system fails. This process, denominated subcritical fracture, has captured the attention of scientists and engineers for more than half a century [2,3], and is considered to be thermally activated (for a review see Ref. [4]). Subcritical fracture of heterogeneous materials is usually studied by two different experimental approaches: acoustic emission (AE) [5-9] or direct imaging (DI) [10-16]. Both AE and DI measurements show that fracture results in a succession of discrete burstlike events (also called jumps or avalanches), whose energies and waiting times follow power law distributions. Although AE detection is an important tool for characterizing rupture, and, for instance, monitoring the progressive damage of civil engineered constructions such as bridges, the extent to which it delivers the same information on the damage amount and dynamics as the one obtained by direct visual inspection is still an open issue. To our knowledge, a comparative analysis between $\mathrm{AE}$ and DI is still lacking, and a combined approach has only been applied to plastic deformation so far [17].

By combining both measurements, this Letter shows that $\mathrm{AE}$ and DI operate at different scales both spatially and temporally. DI is directly associated with the propagation of the fracture, while $\mathrm{AE}$ is related to the rupture process at the scale of the heterogeneities in the material. The damage detected by DI is a consequence of the accumulation of more-elementary events detected by AE. Since it is not possible in our experiment to match $\mathrm{AE}$ and $\mathrm{DI}$ events individually, we use statistics to compare the results obtained by the two measurements. A quantitative agreement is found between the two sequences after the $\mathrm{AE}$ signal has been integrated in time windows corresponding to the temporal resolution of DI. However, we discovered that the acoustic signal analyzed at different time scales produced time scale dependent energy distributions. We argue that correlations between the discrete event acoustic energy and waiting times between events, revealed by the existence of aftershocks, are responsible for the time scale dependent energy distributions. We also discuss why direct imaging will generally not be able to resolve events at a sufficiently small time scale and, thus, will yield a misleading exponent for the energy distribution.

Experimental procedure.-We use fax paper samples from Alrey (as in Refs. [11-13,16]) consisting of a complex network of cellulose fibers with a size distribution between 4 and $50 \mu \mathrm{m}$ and average of $18 \mu \mathrm{m}$ [13]. The samples have a thickness of $50 \mu \mathrm{m}$ and effective dimensions of $21 \times 4 \mathrm{~cm}$, and they are attached to clamping jaws along the longer sides (Fig. 1). In order to control the path and average rupture time of the process, an initial crack of length $\ell_{0}$ is prepared at one of the short free sides of the sample, both in a parallel direction and equidistant from the clamped borders. Experiments are performed by applying a constant force $F$ perpendicularly to the direction of the initial crack. By setting $\ell_{0}=4.75 \mathrm{~cm}$ and $F=200 \mathrm{~N}$, the crack grows, by alternating resting times with fast steps, until reaching a critical length $\ell_{c}=7.8 \pm 0.7 \mathrm{~cm}$ in up to $30 \mathrm{~min}$ after the application of the force. This critical length separates the slow, subcritical rupture dynamics from the catastrophic, quasi-instantaneous one; $\ell_{c}$ is linked to the material toughness, defined by the critical stress intensity factor $K_{c}$ and the applied constant stress $\sigma$ through the relation $K_{c} \sim \sigma \sqrt{\ell_{c}}$. A piezoelectric transducer of diameter $2.3 \mathrm{~mm}$ (Valpey Fisher VP-1.5) is placed in contact with the paper at $5 \mathrm{~cm}$ from the free side containing the initial crack and at $1 \mathrm{~cm}$ from the fixed border (which also corresponds to a $1 \mathrm{~cm}$ distance to 


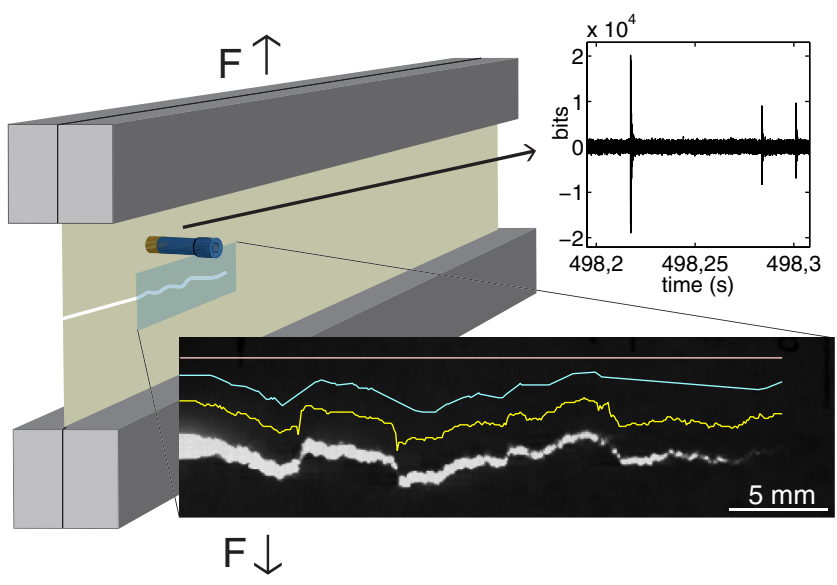

FIG. 1 (color online). Scheme of the experimental setup with typical examples of both an acoustic signal, recorded by a piezoelectric transducer, and an image. The image also shows the paths resulting from adding consecutive crack advances defined as $s_{l}, s_{s}$, and $s$ (from top to bottom).

the initial crack line). An ultrasonic gel guarantees a good contact between the sensor and the sheet of paper. The AE signals are amplified by $64 \mathrm{~dB}$ and recorded continuously during the whole experience by a NI USB-6366 card at $2 \mathrm{MHz}$. A high-speed camera (Photron FASTCAM SA4) takes images in a rectangular area containing the advancing crack at a frequency of $10 \mathrm{~Hz}$ and a spatial resolution of $100 \mu \mathrm{m} /$ pixel. Nine experiments are analyzed, all of them performed under the same conditions. The temperature and relative humidity were $26.5 \pm 1{ }^{\circ} \mathrm{C}$ and $45 \% \pm 2 \%$, respectively. Under these conditions the Young's modulus of the sample is $Y=2.4 \pm 0.2 \mathrm{GPa}$, and its toughness is $K_{c}=5.3 \pm 0.2 \mathrm{MPa} \mathrm{m}^{1 / 2}$.

Data analysis.-In the case of DI, crack contours are extracted using a digital image analysis routine [11]. For each image the position of the crack tip is found. Three variables are defined (see Fig. 1): $s$, the difference in real length of the interface of the fracture between two consecutive images; the size of the jump $s_{s}$, defined as the distance between the crack tip of two successive images; and $s_{l}$, defined as the projection of $s$ on the initial direction of the crack [see its time series in Fig. 2(a)]. For AE, the detection of $\mathrm{AE}$ events is made by calculating the spectral distance $D_{S, N}(t)$, which corresponds to the integral over a time window $w_{d}=0.1 \mathrm{~ms}$ of the difference between the power spectrum of the signal averaged over all the frequencies, $\langle\bar{S}\rangle_{f}(t)$, and the power spectrum of the background noise, averaged over all the frequencies and over a time interval of at least $0.5 \mathrm{~s},\langle\bar{N}\rangle_{f, t}$ (see the Supplemental Material [18]):

$$
D_{S, N}(t)=\frac{1}{w_{d}} \int_{t-\frac{w_{d}}{2}}^{t+\frac{w_{d}}{2}}\left[\langle\bar{S}\rangle_{f}\left(t^{\prime}\right)-\langle\bar{N}\rangle_{f, t}\right] d t^{\prime} .
$$

Acoustic events are detected by thresholding the spectral distance. The threshold is defined as the maximum value of
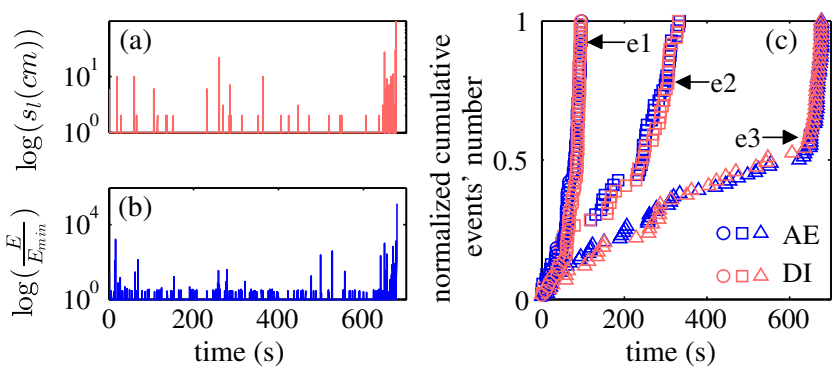

FIG. 2 (color online). Time series of (a) $s_{l}$ and (b) the energy of $\mathrm{AE}$ (normalized to its minimum value $E_{\min }$ ) during one experiment. (c) Comparison between DI activity and AE activity integrated in $0.1 \mathrm{~s}$ time intervals for three experiments $(e 1, e 2$, and $e 3$ ). The experiment represented in (a) and (b) corresponds to experiment 3 in (c).

the spectral distance of the noise with itself. Two main variables are extracted from this analysis: the event energy, calculated as the integral of the spectral distance over the event duration, and the event time, corresponding to the beginning of the event. It is important to point out that with this method we detect almost 4 times more events than by thresholding the signal itself.

Calibration.-The amplitude of the AE signal depends on the contact between the transducer and the sheet of paper, which varies among different realizations. In order to compare the energy of the events from different experiments, a calibration was performed for each realization. It consisted in normalizing the AE signal by the averaged response of the sensor $\left(E_{c}\right)$ to six localized rupture events produced on every sample (by piercing it with a computer controlled thin needle of $250 \mu \mathrm{m}$ of diameter). Also, to evaluate the energy at the source knowing the one measured by the sensor, additional series of experiments were done to study the attenuation of acoustic waves in paper: 10 to 20 localized rupture events were induced on a sheet of paper submitted to a force of $200 \mathrm{~N}$, but with no initial crack to ensure that no uncontrolled rupture would occur. The events were made on a line parallel to the longer sides of the paper, in the same direction as the fracture in the actual experiments. The acoustic signal was recorded by two sensors placed at $4 \mathrm{~cm}$ from each other. By comparing the energy ratio of signals detected by the two sensors to the distance separating each event from the sensors, we obtained that the energy is attenuated following $E(r) / E_{s} \sim(1 / r) \exp \left(-r / r_{c}\right)$, where $E_{s}$ corresponds to the energy at the source $(r \rightarrow 0)$ and $r_{c}$ is a characteristic length equal to $11.1 \mathrm{~cm}$. To determine the position of the source of an acoustic event we use the images and assume that it occurred at the position of the crack tip at the corresponding time. By knowing the distance between the source and the sensor, we can compute the attenuation of the energy. Finally, the energy of an $\mathrm{AE}$ event $E$ [see its time series in Fig. 2(b)] is expressed as $E=E_{s} / E_{c}$. Notice that no echo was measured during the calibration. 
Results.-As the applied force is subcritical and the material heterogeneous, the initial crack propagates in an intermittent manner [11]: images show that the length of the fracture is constant for most of the time and increases by making fast discrete crack steps (jumps). Each jump constitutes a DI event. The acoustic data show discrete bursts with a finite duration between $180-1000 \mu$ s [see inset in Fig. 1]. Each burst constitutes an AE event. First, we compare the number and occurrence times of jumps and AE events, without considering their energy value. Each of the experiments results in approximately 50 jumps and a few hundred acoustic events [Figs. 2(a) and 2(b)], which is a clear indication that the $\mathrm{AE}$ is much more sensitive than the image analysis. Nevertheless, by integrating the energy of the AE signal in consecutive time windows of $w=0.1 \mathrm{~s}$, which corresponds to the temporal resolution of DI, we obtain a new time series denominated $\mathcal{E}\left(t_{w}\right)$ where $t_{w}$ corresponds to the starting time of each window. Each nonzero value of $\mathcal{E}(t)$ constitutes an integrated event. Figure 2(c) shows that the activity in terms of cumulative event number is very similar for $\mathrm{DI}(\mathrm{t})$ and $\mathcal{E}(t)$. The number of $\mathrm{AE}$ events to number of DI events ratio is equal to $1.02,1.13$, and 1.54 for $e 1, e 2$, and $e 3$ in Fig. 2(c) respectively, and $1.07 \pm 0.31$ when we consider all the experiments. The dispersion of these coefficients may be associated to the variations in the contact between the transducer and the paper; however, the fact that they are of order 1 suggests that $\mathrm{AE}$ delivers the same information provided it is analyzed at the same time scale as DI, and, in particular, that the number of DI events becomes equivalent to the number of integrated AE events. From the raw acoustic signal, up to 10 times more $\mathrm{AE}$ events can be detected than by DI. We verified that this is not due to the lower time scale of the DI analysis. Indeed, a higher image acquisition rate $(2000 \mathrm{~Hz})$ was unable to detect the more-elementary events that are captured by the acoustics. Therefore, acoustics is a technique that may be able to detect events down to the fiber scale while DI requires the accumulation of enough elementary rupture events able to produce an observable damage.

The temporal correlation between $s_{l}(t)$ and $\mathcal{E}(t)$ is very weak, which makes it impossible to match jumps and acoustic events individually. However, our data are suitable for statistical analysis. Here, we will study and compare the probability distributions of two different variables characterizing subcritical fracture: waiting times and energies.

Waiting times:-For subcritical fractures the time between two discrete events often follows power-law distributions [7,15,19]. Figure 3 shows the probability distributions of waiting times between the events for the jumps and the AE data for all the experiments combined. The distributions were fit as power laws and the obtained exponents are very similar: $1.06 \pm 0.05$ and $1.00 \pm 0.03$ for the jumps and AE waiting times, respectively.

Since acoustic data acquisition has a much better time resolution, acoustic waiting times spread on a larger set of values. However, by adjusting the distribution
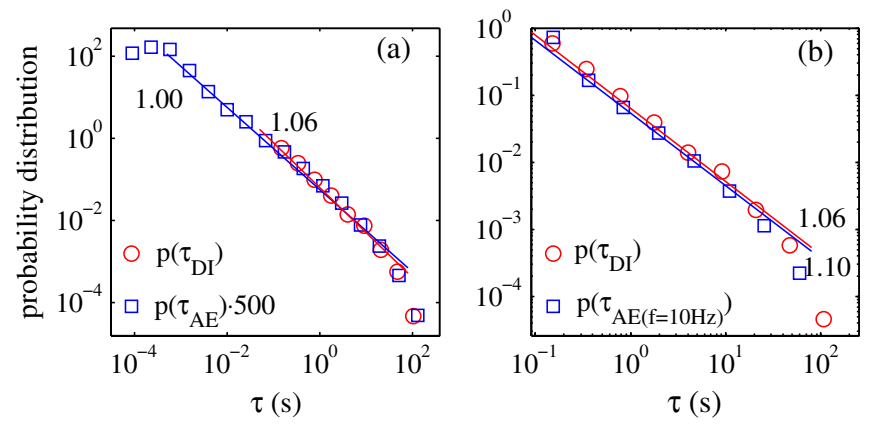

FIG. 3 (color online). (a) Distribution of waiting times of the jumps, obtained by DI (red circles) and distribution of waiting times of AE multiplied by 500 (blue squares). (b) Distribution of waiting times obtained by DI (red circles) and distribution of waiting times of $\mathcal{E}$, obtained by integrating $E$ in $0.1 \mathrm{~s}$ time intervals (blue squares).

normalization coefficients, the distributions collapse [Fig. 3(a)]. Distributions also match, without any adjustments, when analyzing the waiting time of $\mathcal{E}(t)$ [Fig. 3(b)]. The overall similitude between the two probability distributions indicates that all the acoustic bursts may correspond to fracture events.

Energies:-In this two-dimensional system, fracture energy scales to first order as the crack length. Therefore, the normalized distribution of jump sizes is also equal to the normalized distribution of jump energies. The probability distributions of $s, s_{s}$, and $s_{l}$ are represented in Fig. 4. The three distributions follow a power law over approximately one decade followed by a cutoff. Because of the relatively small number of jumps and the limited span of the power law, we based our analysis on the similar shape of the distributions as compared to the one reported in a previous work, under similar conditions, where an

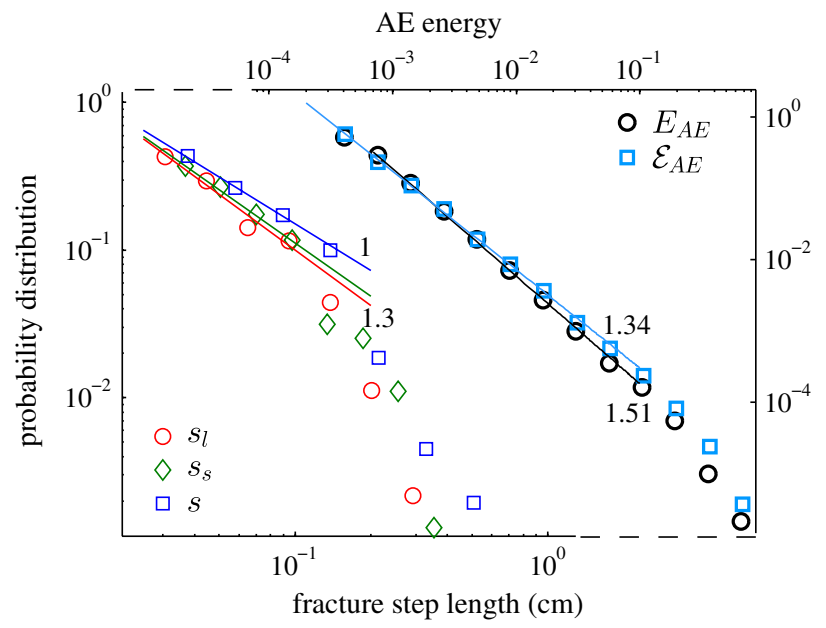

FIG. 4 (color online). Left: probability distribution of fracture jumps $\left(s_{l}, s_{s}\right.$, and $\left.s\right)$. Right: probability distributions of acoustic event energy at its original frequency, $E$ (black circles). and after integrating over $0.1 \mathrm{~s}$ time intervals, $\mathcal{E}$ (blue squares). The values correspond to the exponents of the power laws. 
(a) $\beta$ variation

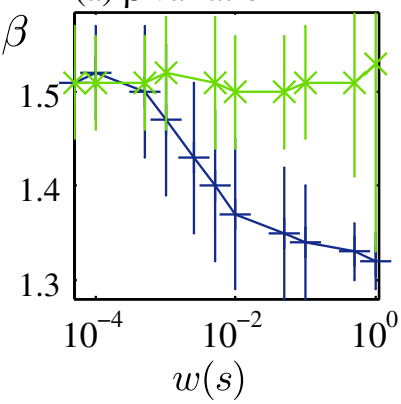

(d) uncorrelated wt

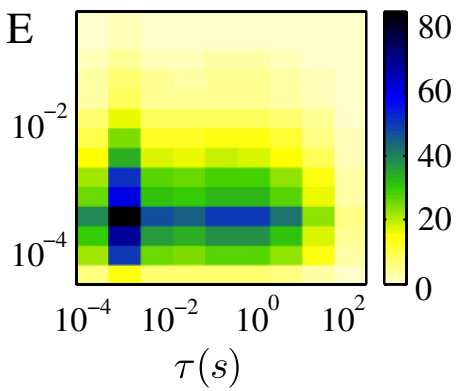

(b) wt before

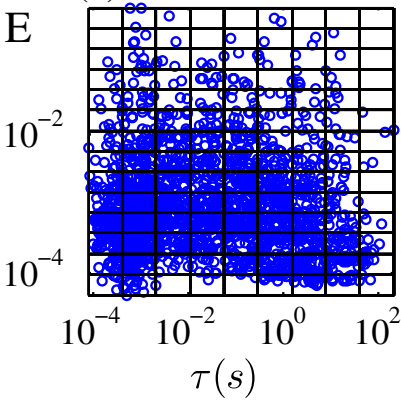

(e) $(\mathrm{B}-\mathrm{D}) / \mathrm{D}$

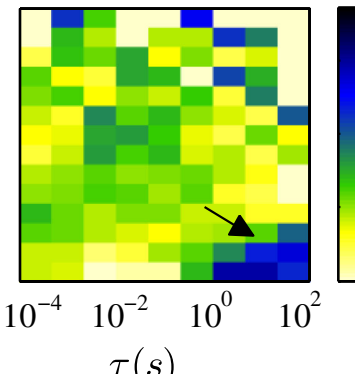

(c) wt after

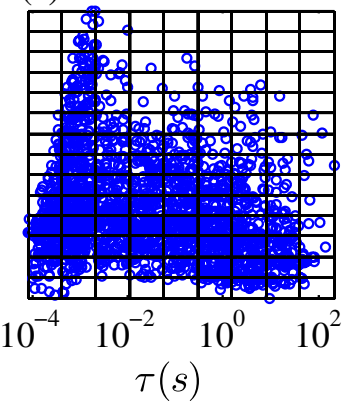

(f) $(\mathrm{A}-\mathrm{D}) / \mathrm{D}$

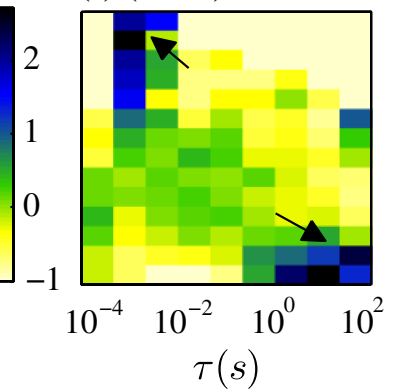

FIG. 5 (color online). (a) Variations of the exponent $\beta$ with the analysis time window $w$. For the actual data $\beta$ decays, while it remains constant for the artificial uncorrelated case. (b) Repartition of event energies and waiting times before events. (c) Repartition of event energies and waiting times after events. (d) Repartition of event energies and mixed-up waiting times. (e) Relative difference between the number of events in each interval for waiting times before the event and decorrelated waiting times. (f) Relative difference between the number of events in each interval for waiting times after the event and decorrelated waiting times.

exponent of 1.23 has been found for the power law distribution of $s_{l}$ [12]. By fitting the power laws we extracted an exponent $1.3 \pm 0.2$ for $s_{s}, 1.3 \pm 0.2$ for $s_{l}$, and $1 \pm 0.1$ for $s$. These values are coherent among themselves (smaller value for $s$, due to its fractal structure) as well as with the previous work (see the Supplemental Material [18] and Ref. [12]), which presented a much better statistics.

The estimated acoustic energy $E$ follows a power law with an exponent $\beta=1.51 \pm 0.06$ (notice that a value of 3 / 2 has been predicted by an analytical model [11]), which is significantly different from the exponent of the power law distribution of jumps. Now, if we look at the distribution of acoustic energy $\mathcal{E}$, which has been obtained at the same time scale as the crack jumps from the images, we find a power law over more than three decades with an exponent $\beta=1.34 \pm 0.08$, closer to the one found for the distributions of jump sizes (Fig. 4). This similarity between DI and $\mathrm{AE}$ has been verified at larger time windows $(0.2 \mathrm{~s}, 0.5 \mathrm{~s}$, and $1 \mathrm{~s}$ ). If we do the same analysis for time windows $0.1 \mathrm{~ms}<w<1 \mathrm{~s}$, we find an exponent $\beta$ that varies continuously but with an abrupt change around a time scale of $1 \mathrm{~ms}$ [Fig. 5(a)]. The strong dependence of $\beta$ on $w$ may be resulting from temporal correlations, linking small waiting times with high energy events. Indeed, autocorrelation of the AE signal shows the existence of temporal correlations at the same time scale. We have verified that this correlation corresponds to actual aftershocks and is not an artifact linked to the duration of the events.

Aftershocks.-To understand what this correlation time corresponds to, we study waiting times and energies of the events of all the experiments combined. In Figs. 5(b) and 5(c) each event is represented by a point on an energywaiting time diagram. Each event is defined by its energy and can be represented with [Fig. 5(b)] the waiting time that precedes it or [Fig. 5(c)] the waiting time that follows it. We can see in Fig. 5(c) that there is a large density of events for large energies (top) and small waiting times (left). To compare this to a situation where waiting times and energies are uncorrelated, we construct an artificial time series in which we destroy correlations by keeping the same AE event arrival times, but redistributing randomly the values of the $\mathrm{AE}$ event energies. The three diagrams are divided in $14 \times 9$ cells, and the number of events in each cell is stored in $14 \times 9$ matrices: $B$ for waiting times before the events, $A$ for waiting times after the events, and $D$ for the uncorrelated waiting times obtained, from more accuracy, as an average over an ensemble of 1000 artificial time series [Fig. 5(d)]. Finally, a comparison between the experimental results $(A$ and $B)$ and the uncorrelated case $(D)$ is made by calculating the relative difference between the matrices. The result is shown in Figs. 5(e) and 5(f). We find that the number of events having large energy and followed by small waiting times (aftershocks) is 
significantly larger for the experimental data than for the uncorrelated case [top arrow in Fig. 5(f)]. On the other hand, this behavior is not observed for waiting times preceding the events (foreshocks) [Fig. 5(e)]. This fact shows the existence of aftershocks during the propagation of the crack, with a typical waiting time of $10^{-3} \mathrm{~s}$. Also, for both waiting times (before and after the event) we observe a large density for small energies [arrows at bottom right of Figs. 5(e) and $5(\mathrm{f})]$, meaning that there exist "inactivity times" characterized by long waiting times and low energy events.

Finally, we find that analyzing the energy distribution of the artificial $\mathrm{AE}$ time series at time windows from 0.1 to $100 \mathrm{~ms}$ does not change the value of the exponent [Fig. 5(a)]. This confirms that the temporal correlations are responsible for the change in the exponent obtained when analyzing the experimental AE signals at different time scales.

Conclusion.-We studied the subcritical crack growth of a single crack in a sheet of paper submitted to a constant force. By combining DI and AE measurements, this Letter shows that the two methods operate at different scales both spatially and temporally: acoustics captures fast rupture events at the scale of the fibers, while the accumulation of these events provokes a damage zone that will eventually be detected by the image analysis. Besides these differences, $\mathrm{DI}$ and $\mathrm{AE}$ yield a statistical similitude between them concerning the distribution of waiting times and the distribution of event energies, provided $\mathrm{AE}$ is analyzed at large time scales corresponding to the image acquisition rate.

Temporal correlations at a typical scale of $1 \mathrm{~ms}$ in the form of aftershocks have been observed on the acoustic signal. These correlations makes the energy probability distribution very sensitive to the time scale at which measurements are done. DI, which is unable to resolve those aftershocks, leads to a similar exponent value as acoustics analyzed at a time scale larger than the correlation time.

This exponent, often analyzed in the case of earthquakes [20], is the most important variable that characterizes a scale invariant dynamics. It is related to the existence or absence of criticality in the dynamics [21] and also to the predictability of catastrophic events [22]. In addition, it is often the criterion for discriminating between different models that try to explain their avalanche dynamics (fiber bundles [23,24], percolation [25], elastic lines in disordered media [26,27], self-organized criticality [28], etc.) and eventually to classify it into different universality classes. By showing that low frequency monitoring of the rupture dynamics unable to resolve aftershocks results in a misleading value of the measured exponent, our work emphasizes the importance of the choice of the experimental method in order to characterize the subcritical crack growth in heterogeneous materials, but also in the general frame of scale-invariant avalanches.

We acknowledge R. Planet for helping in the automatization of the experiment and the Federation of Research "A. M. Ampère" of Lyon (FRAMA) for financial support.
* Corresponding author. osvanny.ramos@univ-lyon1.fr

[1] A. A. Griffith, Phil. Trans. R. Soc. A 221, 163 (1921).

[2] S. S. Brenner, J. Appl. Phys. 33, 33 (1962).

[3] S. N. Zhurkov, Int. J. Fract. Mech. 1, 311 (1965); reprinted in Int. J. Fract. 26, 295 (1984).

[4] L. Vanel, S. Ciliberto, P.-P. Cortet, and S. Santucci, J. Phys. D 42, 214007 (2009).

[5] A. Petri, G. Papro, A. Vespignani, A. Alippi, and M. Constantini, Phys. Rev. Lett. 73, 3423 (1994).

[6] A. Garcimartín, A. Guarino, L. Bellon, and S. Ciliberto, Phys. Rev. Lett. 79, 3202 (1997).

[7] S. Deschanel, L. Vanel, N. Godin, G. Vigier, and S. Ciliberto, J. Stat. Mech. (2009) P01018.

[8] L. I. Salminen, A. I. Tolvanen, and M. J. Alava, Phys. Rev. Lett. 89, 185503 (2002).

[9] J. Rosti, J. Koivisto, and M. J. Alava, J. Stat. Mech. (2010) P02016.

[10] T. Baumberger, C. Caroli, and D. Martina, Nat. Mater. 5, 552 (2006).

[11] S. Santucci, L. Vanel, and S. Ciliberto, Phys. Rev. Lett. 93, 095505 (2004).

[12] S. Santucci, P.-P. Cortet, L. Vanel, and S. Ciliberto, Proceedings of the 11th International Congress on Fracture (ICF11), Turin, Italy, 2005 (Curran Associates, New York, 2010), Vol. 7, p. 5432.

[13] S. Santucci, P.-P. Cortet, S. Deschanel, L. Vanel, and S. Ciliberto, Europhys. Lett. 74, 595 (2006).

[14] K. J. Måløy, S. Santucci, J. Schmittbuhl, and R. Toussaint, Phys. Rev. Lett. 96, 045501 (2006).

[15] S. Santucci, L. Vanel, and S. Ciliberto, Eur. Phys. J. Spec. Top. 146, 341 (2007).

[16] O. Ramos, P.-P. Cortet, S. Ciliberto, and L. Vanel, Phys. Rev. Lett. 110, 165506 (2013).

[17] J. Weiss, T. Richeton, F. Louchet, F. Chmelik, P. Dobron, D. Entemeyer, M. Lebyodkin, T. Lebedkina, C. Fressengeas, and R. McDonald, Phys. Rev. B 76, 224110 (2007).

[18] See Supplemental Material at http://link.aps.org/ supplemental/10.1103/PhysRevLett.112.115502 for detailed information.

[19] F. Kun, Z. Halász, J. S. Andrade, Jr., and H. J. Herrmann, J. Stat. Mech. (2009) P01021.

[20] D. Schorlemmer, S. Wiemer, and M. Wyss, Nature (London) 437, 539 (2005).

[21] J. X. de Carvalho and C. P. C. Prado, Phys. Rev. Lett. 84, 4006 (2000).

[22] O. Ramos, Tectonophysics 485, 321 (2010).

[23] S. Pradhan, A. Hansen, and B. K. Chakrabarti, Rev. Mod. Phys. 82, 499 (2010).

[24] Z. Danku and F. Kun, Sci. Rep. 3, 2688 (2013).

[25] A. Hansen and J. Schmittbuhl, Phys. Rev. Lett. 90, 045504 (2003).

[26] D. Bonamy and E. Bouchaud, Phys. Rep. 498, 1 (2011).

[27] P. Le Doussal and K. J. Wiese, Phys. Rev. E 88, 022106 (2013).

[28] A. Vespignani and S. Zapperi, Phys. Rev. E 57, 6345 (1998). 